\title{
Development of a piezoelectric multi-axis stage based on stick-and-clamping actuation technology
}

\author{
Sang Won Lee ${ }^{1,4}$, Kyoung-Gee $\mathrm{Ahn}^{2}$ and Jun $\mathrm{Ni}^{3}$ \\ ${ }^{1}$ School of Mechanical Engineering, Sungkyunkwan University, 300 Cheoncheon-dong, \\ Jangan-gu, Suwon 440-746, Korea \\ ${ }^{2}$ Computopia, 205 Hallene Road, Warwick, RI 02886, USA \\ ${ }^{3}$ S M Wu Manufacturing Research Center, University of Michigan, 2350 Hayward Street, \\ Ann Arbor, MI 48109, USA \\ E-mail: sangwonl@skku.edu
}

Received 30 March 2007, in final form 2 September 2007

Published 12 October 2007

Online at stacks.iop.org/SMS/16/2354

\begin{abstract}
This paper presents the design, analysis and fabrication of a piezoelectric multi-axis stage based on a new stick-and-clamping actuation technology for miniaturized machine tool systems, referred to as meso-scale machine tool (mMT) systems. In the stick-and-clamping actuation system,

shearing/expanding piezoelectric actuators, an inertial mass and an advanced preload system are configured innovatively to generate the motion of an inertial mass. There are two operating modes in the stick-and-clamping actuation technology: (1) stick mode and (2) clamp mode. In stick mode, the 'slow' deformation of the shearing piezoelectric actuators drives an inertial mass, which is located on the tips of the shearing piezoelectric actuators, by means of the friction force at their contact interface. On the other hand, in clamp mode, the expanding piezoelectric actuators provide the clamping force to an inertial mass when the rapid backward deformation of the shearing piezoelectric actuators occurs. The stick-and-clamping actuation technology also enables two-degrees-of-freedom (DOF) motion of an inertial mass in a single plane by perpendicularly stacking two shearing piezoelectric actuators. The 2-DOF piezoelectric multi-axis stage is developed on the basis of the stick-and-clamping actuation technology, and the dynamic and static performance analyses are conducted. The LuGre friction model for the contact interfaces is introduced, and their dynamic behaviours are characterized. In the open-loop static performance test, linear, diagonal and circular motions of the developed piezoelectric multi-axis stage are generated, and their performances are evaluated. The dynamic characteristics and static performances of the developed 2-DOF piezoelectric multi-axis stage show its applicability and effectiveness for the precision positioning system.
\end{abstract}

(Some figures in this article are in colour only in the electronic version)

\section{Introduction}

Miniaturized machine tool systems, referred to henceforth as meso-scale machine tool (mMT) systems, have recently been

4 Author to whom any correspondence should be addressed. proposed as a way to replace the MEMS-based technologies for the manufacture of micro/meso-scale components having complex geometries with a broad range of materials [1]. To effectively produce micro/meso-scale components, the mMT should be successfully developed through innovative 
miniaturization of its subsystems and their appropriate integration.

The positioning system is a crucial subsystem in a machine tool since it facilitates the relative motion between a workpiece and a tool for the completion of the machining processes. Conventional machine tools have generally adopted lead screw or ball screw technology to realize their positioning systems. Several one-degree-of-freedom (DOF) stages based on screw technology can be properly stacked together to obtain the multi-DOF relative motion between a workpiece and a tool. Similarly, the mMT also requires a precision positioning system with a reduced size. However, the miniaturization of a conventional screw technology-based stage is challenging due to its manufacturing difficulty and degradation of accuracy. Therefore, the piezoelectric inchworm actuation system has drawn significant attention as an alternative technology to effectively develop the miniaturized positioning system for the mMT due to its slim profile, large displacement, low energy consumption, high resolution and high positioning accuracy.

The piezoelectric inchworm devices are generally categorized into one of three basic groups. The first group involves a 'walker' configuration, in which the piezoelectric elements move with a shaft; the second basic configuration is the 'pusher' in which the piezoelectric elements push the shaft with an inchworm technique. The third group is a combination of walker and pusher. In this arrangement, the piezoelectric elements are not completely removed from the actuation shaft

Stibitz was among the first to design an effective inchworm actuation technique [2]. A typical pusher method incorporating magnetostrictive actuators was used to drive a shaft in their design. The focus of their design was to create a positioning actuator for use in precision machining and tooling. In the late 1960s, several positive improvements in piezoelectric inchworm actuators were achieved. Hsu et al introduced the first patented piezoceramic inchworm actuator in 1966 and their design involved a single hollow cylindrical element to clamp and extend with a walker configuration [3]. Locher designed the inchworm actuator which was configured in a hybrid structure with the clamping elements engaging a 'clam-claw' mechanism, resulting in an individual step as small as $12.7 \mu \mathrm{m}$ [4]. This method was the first hybrid inchworm actuator which applied a maximum holding force in the inactivated state. Brisbane developed the first walker-type piezoceramic inchworm actuator, and the incremental step size was down to $5 \mu \mathrm{m}$ in 1968 [5]. The inchworm actuator which was developed by Galutva in 1972 was the first to use stacked piezoceramic elements to increase the actuation length [6]. These stacked piezoelectric actuators were configured in a walker fashion both to generate forward motion and to directly clamp the walls of the guided channel.

The most important and first piezoelectric pusher-type inchworm actuator was designed by Bizzigotti [7] and May [8] in 1975. In Bizzigotti and May's inchworm design, the Ushaped piezoelectric elements clamped a shaft and extended, which is similar to the recent inchworm actuation manner, and the incremental step size was down to $0.004 \mu \mathrm{m}$. They first introduced the term 'inchworm' to describe such devices. EXFO Burleigh Products Group, formerly Burleigh Instruments Inc., has successfully developed commercial inchworm systems for more than 30 years based on these patents [9]. O'Neill and Foster presented the walker-type inchworm actuator that consisted of two clamping discs coupled with 'cups' in 1980 [10]. These 'cups' pushed against the cylinder wall when zero voltage was applied and they retracted from the wall when the discs were actuated. As a result, the stacked piezoelectric discs were extended.

Taniguchi presented the piezoelectric actuator that acted like an earthworm rather than an inchworm by stacking multiple piezoceramic elements in 1984 [11]. These elements generated both clamping and extending actions, resulting in a wave-like or peristaltic motion. The pusher-type inchworm actuator developed by Staufenberg used elliptic motion of the piezoelectric actuators and was capable of producing either translation or rotation [12]. Fujimoto developed a walker-type piezoelectric motor in 1988, and its most important idea was using the structural flexures to create the clamping action [13]. The structural flexures allowed an amplified displacement, resulting in more flexibility of the machining tolerances.

In 1990, Murata developed a novel pusher-type inchworm actuator including a linear gear rack with extremely small pitch that was driven by a set of piezoceramic elements [14]. In this design, the position of the gear rack was controlled to engage or disengage a shaft, and another set of piezoceramic elements generated the motion of a shaft. Rennex presented a novel arrangement of piezoelectric elements in a parallel manner with hybrid pusher-walker actuation in 1994, resulting in a considerably slimmer profile [15]. This method was similar to Locher's design, but the 'clam-claw' mechanism was replaced with a flexural clamp. Miesner and Tester presented the inchworm actuator using a hybrid TERFENOL$\mathrm{D}$ and piezoceramic elements in 1994 [16]. Their pushertype design used TERFENOL-D magnetostrictive elements for extension and the piezoceramic for clamping, and was capable of producing a stall force of $116 \mathrm{~N}$ and a rate of $25.4 \mathrm{~mm} \mathrm{~s}^{-1}$ without load. However, the size of their design was rather large, spanning almost $150 \mathrm{~mm}$, and the resonant drive electronics were also of attention. Lee et al proposed an inchworm actuator fabricated by the semiconductor manufacturing technique [17]. This design was not very different from previous ones, but the size was considerably reduced.

In 1996, Pandell and Garcia developed a piezoceramic caterpillar motor, which resembled that of Galutva, but the focus had shifted to larger forces and displacements by adding one extra stage for extension and clamping [18]. Galante proposed a linear piezoelectric inchworm motor with large displacement range $(6 \mathrm{~mm})$ and large holding force capability (200 N) [19]. In his pusher-type design, the inchworm motion was completed by using three active piezoceramic elements: two clamps and one pusher. The sequential motions of the piezoceramic elements generated the forward motion of the inner shaft with respect to the outer housing.

Canfield et al developed a millimeter-scale piezoelectric inchworm actuator for use with a compliant end effector in minimally invasive surgery (MIS) [20]. Their design used a flexure machined from titanium by the wire electrical discharge machining (EDM) process, and the piezoelectric inchworm moved back and forth to generate the motion of jaws of the end effector, resulting in its gripping and releasing. Kim et al modified Meisner and Tester's hybrid 
inchworm design by adding a flexure structure in 2002 [21]. Their design ensured more secure clamping action with multistack piezoelectric actuators and the flexure, resulting in high push force and resolution with long traveling distance. Suleman et al presented an electrostrictive inchworm actuator with a large displacement and medium force rating [22]. Their design consisted of two flex-tensional brake assemblies and one central electrostrictive stack located between two brake assemblies to realize 'walker' motion of the shaft. The brake assembly consisted of the flexural frame and electrostrictive stack and provided the clamping action with no electrical power. With the activation of the electrostrictive stack in the brake assembly, the shaft was unclamped and 'walked' with the extension of the central electrostrictive stack. In 2005, Li et al developed the pusher-type piezoelectric inchworm actuator with two flexural clamps operated by tubular piezoelectric stacks and one extensional piezoelectric stack for 'pushing' [23]. This design was a reversal of that of Suleman et al in terms of an inchworm mechanism. The tubular piezoelectric stacks in the flexural clamp provided clamping and unclamping actions to the shaft, and the motion of the shaft was obtained by the 'pushing' of the extensional piezoelectric stack. The walker-type piezoelectric inchworm actuator was proposed by Kim et al by introducing arc shell structures for the clamping mechanism in 2005 [24]. Their design used three identical 'cells' and each cell was composed of an arc shell structure and a piezoelectric stack actuator, resulting in structural simplification. These three 'cells' were connected together in series, and the sequential extension and contraction of the piezoelectric stacks realized a 'walking' motion and clamping inside the circular casing.

The inchworm actuators described above have shown significant technological advancement in terms of positioning accuracy, resolution, driving mechanism, displacement, velocity, force and size. As a result, they have been successfully applied to the precision positioning system in various fields such as optics, robotics, machining, semiconductor manufacturing, medicine, measurement, and so forth. However, these inchworm devices could provide only 1-DOF motion and it is, therefore, necessary to stack several linear or rotary stages to obtain multi-DOF positioning. In addition, stacking stages can give rise to potential assembly error and increased size.

The stick-and-slip actuator that could be categorized into a pusher-type inchworm actuator was introduced by Breguet et $a l$ and can be a potential technique for the miniaturized positioning system of the mMT due to its capability of producing in-plane 2-DOF motion [25-27]. The shearing piezoelectric actuators had contacts with an inertial mass and the friction force at their contact interfaces generated the motion of an inertial mass according to the deformation of the shearing piezoelectric actuators. A magnetic force was used to apply a normal force to the contact interfaces. The stick-and-slip actuation technology can also generate inplane 2-DOF motion of an inertial mass by assembling two shearing piezoelectric actuators in a perpendicular fashion. Its ability to obtain 2-DOF motion in a single plane could be advantageous for miniaturization of the positioning system and may eliminate potential assembly errors that may occur in stacked stages. However, the 2-DOF stick-and-slip actuation system suffered from backward motion of an inertial mass in slip mode and its unwanted rotational motion.

A new actuation technology, referred to as stickand-clamping actuation technology, will be proposed and implemented in a novel multi-axis positioning system for the $\mathrm{mMT}$ in this paper. The proposed stick-and-clamping actuation technology incorporates shearing/expanding piezoelectric actuators, an inertial mass and an advanced preload system, and will overcome the limitations of the stick-and-slip actuation technology. The configuration and operating principle of the stick-and-clamping actuation technology are stated in section 2 and the innovative design and fabrication of the proof-of-concept prototype of the multi-axis stage will follow. Performance evaluations on the developed multiaxis stage will also be provided through dynamic and static performance analyses. In dynamic analysis, the friction model based on the LuGre approach will be proposed for the contact interfaces and its equivalent parameters will be identified to characterize the dynamic behavior of the stickand-clamping actuation system. Static performance analysis, including linear, diagonal and circular motion tests, will show the effectiveness and applicability of the developed multi-axis stage for the positioning system of the mMT.

\section{Operating principle}

\subsection{Stick-and-slip actuation}

Stick-and-slip actuation technology utilizes the deformation of shearing piezoelectric actuators to generate the motion of an inertial mass [25]. The shearing piezoelectric actuator deforms in a shear direction when an electrical field is applied. The deformation of the shearing piezoelectric actuator $(\delta)$ can be simply calculated by equation (1) when a voltage input is applied:

$$
\delta=d_{15} V
$$

where $d_{15}$ is a piezoelectric shear mode coefficient and $V$ an applied voltage.

This shear deformation of the shearing piezoelectric actuator is a key input for driving an inertial mass in the stickand-slip actuation system. Figure 1 illustrates the operating principle of the stick-and-slip actuation technology. The motion of an inertial mass, denoted by slider in figure 1 , can be generated by applying a sawtooth voltage input to the shearing piezoelectric actuators. A hemispherical tip is attached to the shearing piezoelectric actuator to provide contact interface with the slider. In addition, a permanent magnet is located under the slider to provide normal force to the contact region. There are two modes in the stick-and-slip actuation technology: (1) stick mode and (2) slip mode.

The stick mode occurs at the increasing ramp portion of the sawtooth voltage input. In stick mode, the deformation of the shearing piezoelectric actuators occurs 'slowly' and generates the motion of the slider owing to the friction at its contact interfaces with the tips of the shearing piezoelectric actuators. In slip mode, however, the sawtooth voltage input drops suddenly from positive to negative values and the slider does not follow ideally the deformation of the shearing piezoelectric actuators due to its inertia. However, in reality, the perfect slip does not exist and the slight backward motion 
Development of a piezoelectric multi-axis stage based on stick-and-clamping actuation technology

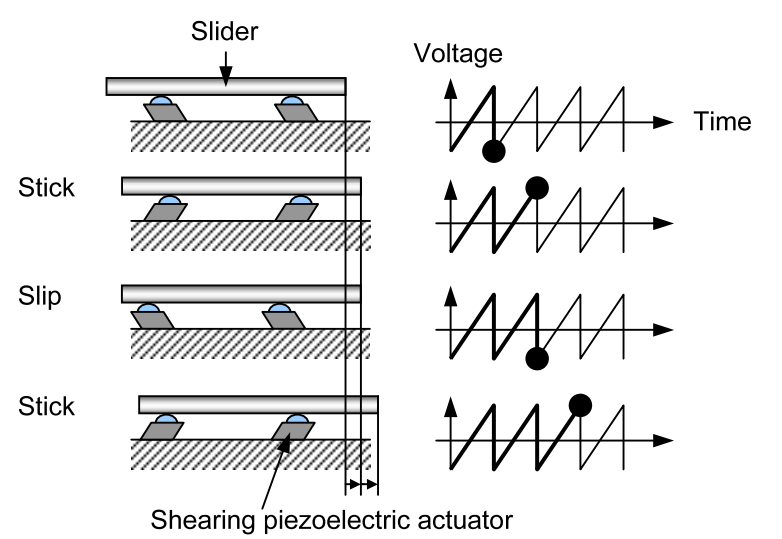

Figure 1. Operating principle of the stick-and-slip actuation technology.

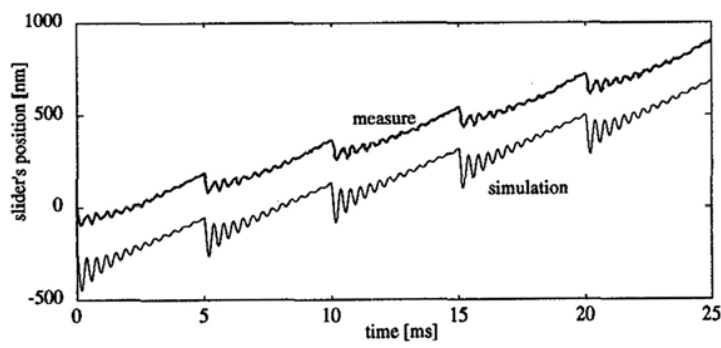

Figure 2. Measured and simulated displacements of slider in stick-and-slip actuation system [25] (ㄷ 1998 IEEE). The slight backward motion and the residual vibration of the slider appeared in both measured and simulated results.

of the slider following the rapid backward deformation of the shearing piezoelectric actuators is monitored. Figure 2 shows an example of the backward motion of the slider in the stick- and-slip actuation system [25]. This backward motion of the slider in slip mode can hamper the direct application of the stick-and-slip actuation technology to the precision machining process, and must be properly compensated for.

\subsection{Stick-and-clamping actuation}

A new stick-and-clamping actuation technology is proposed by adding expanding piezoelectric actuators and an advanced preload system to the stick-and-slip actuation technology to overcome its limitations. The operating principle of the proposed stick-and-clamping actuation technology is illustrated in figure 3. As can be seen in figure 3, sawtooth and pulse voltage signals are used to activate shearing and expanding piezoelectric actuators, respectively.

The stick-and-clamping actuation technology includes two operating modes: (1) stick mode and (2) clamp mode. In stick mode, similar to the case of the stick-and-slip actuation technology, the ramp portion of the sawtooth voltage input generates the motion of the slider by the friction force at its contact interfaces. On the other hand, in clamp mode, when the sawtooth voltage input suddenly drops, the pulse voltage signal is applied to the expanding piezoelectric actuators. Thus, they elongate and provide gripping forces to the slider when the shearing piezoelectric actuators deform rapidly in the backward direction. As a result, the backward motion of the slider can be eliminated. Continuous motion of the slider can be obtained through repetitive stick and clamping actions.

In contrast to the magnetic preload system in the stick-and-slip actuation technology, the stick-and-clamping actuation technology has an improved preload system that is realized by spring ball plungers and a thrust bearing. Therefore, it is possible to precisely control the normal and friction forces at each contact interface between shearing

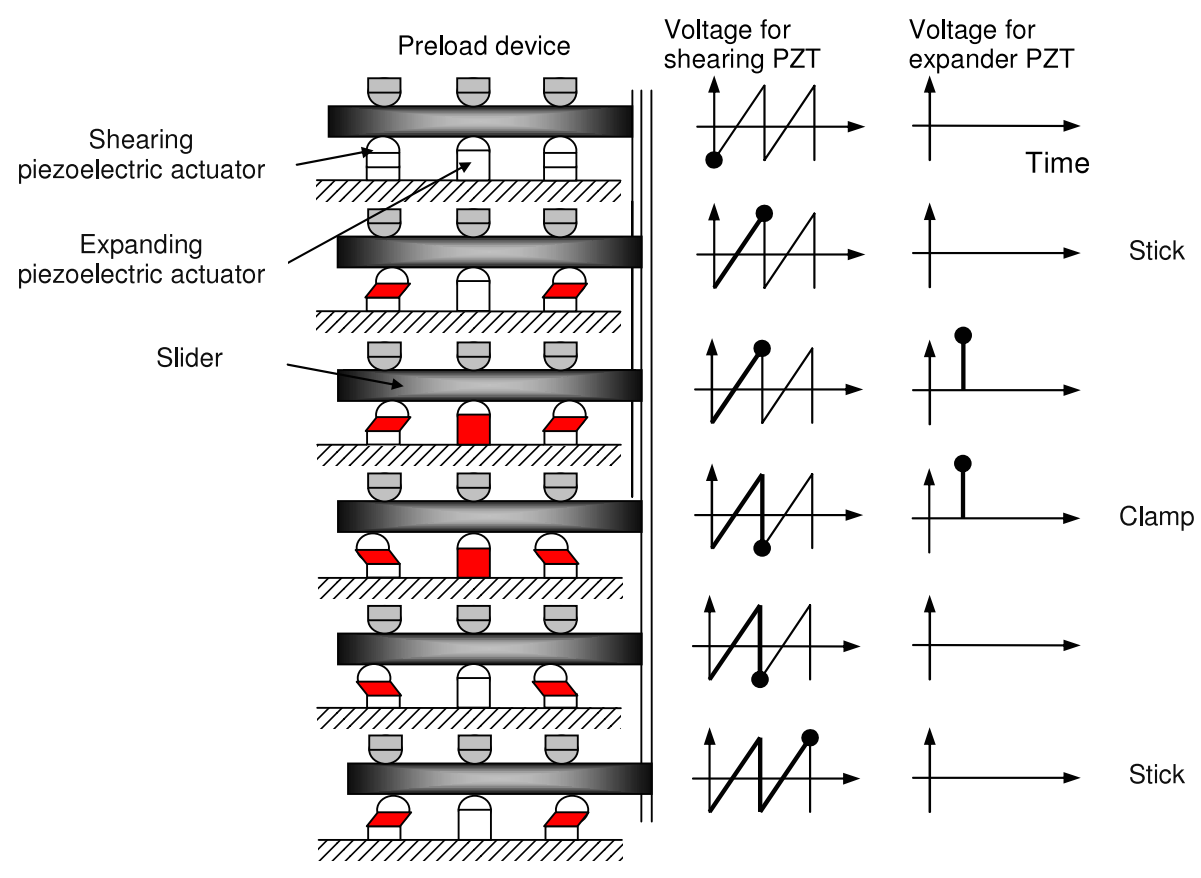

Figure 3. Operating principle of the stick-and-clamping actuation technology. The shaded actuators represent their activated state. 


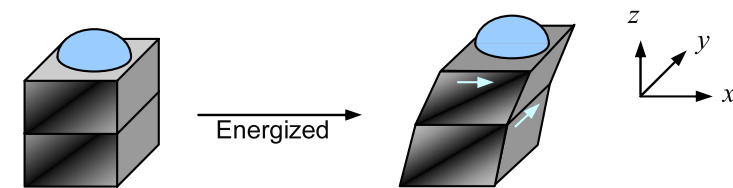

Figure 4. Schematic diagram of the two-mode shearing piezoelectric actuator.

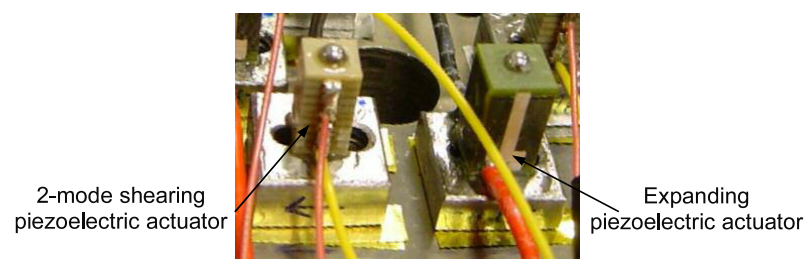

Figure 5. Photo of the two-mode shearing and expanding piezoelectric actuators. The sizes of the two-mode shearing and expanding piezoelectric actuators are $4.11 \mathrm{~mm} \times 4.23 \mathrm{~mm}$ $\times 8.92 \mathrm{~mm}$ and $5.12 \mathrm{~mm} \times 5.21 \mathrm{~mm} \times 10.07 \mathrm{~mm}$, respectively. The material of piezoelectric elements utilized in both actuators is PIC-255 and the hemispherical tips made by SS440C stainless steel are attached to the tops of both actuators. The diameter of the hemisphere tip was $1 \mathrm{~mm}$. The capacitance of each actuator perpendicularly stacked in the two-mode shearing piezoelectric actuator was $1.8 \mathrm{nF}$ and that of the expanding piezoelectric actuator was $0.7 \mu \mathrm{F}$.

piezoelectric actuators and the slider, resulting in the enhancement in the accuracy of the motion of the slider.

The stick-and-clamping actuation technology can also be extended to obtain the 2-DOF motion of the slider in a single plane. Two shearing piezoelectric actuators are stacked together in a perpendicular fashion, and their simultaneous deformations in two perpendicular directions allow the in-plane 2-DOF motion of the slider. Figure 4 illustrates the schematic diagram of the stacked shearing piezoelectric actuator, henceforth referred to as the two-mode shearing piezoelectric actuator, whose poling directions are perpendicular to each other.

\section{Design and fabrication of the piezoelectric multi-axis stage}

\subsection{Piezoelectric actuators}

In order to effectively implement the proposed stick-andclamping actuation technology into the positioning system of the mMT, the speed of the slider depending upon the deformation rate of the shearing piezoelectric actuators should be an order of a few millimeters per second. In general, the deformation of a single shearing piezoelectric element is very small, and the magnitude and frequency of sawtooth voltage input should be increased to obtain the higher deformation rate. However, the only electrical amplification is not sufficient to obtain the desired deformation rate of the shearing piezoelectric actuator due to its limitations of maximum voltage input and frequency. Therefore, mechanical amplification was also carried out to increase the deformation rate of the shearing piezoelectric actuator by stacking several thin shearing piezoelectric elements. The actual shearing piezoelectric actuator was fabricated by stacking four

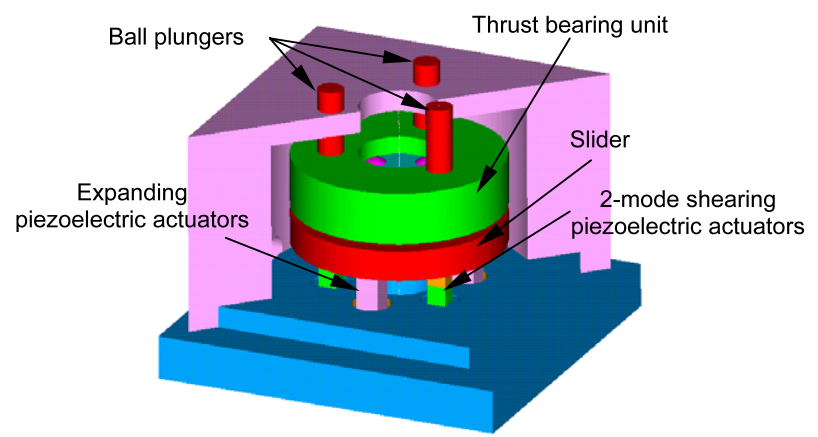

Figure 6. Solid model of the prototype of the 2-DOF piezoelectric multi-axis stage.

thin shearing piezoelectric elements, and therefore its total deformation was four times larger than that of a single shearing piezoelectric element. Two shearing piezoelectric actuators were stacked together in a perpendicular fashion to complete the two-mode shearing piezoelectric actuator. The expanding piezoelectric actuator was also fabricated with multilayer piezoelectric ceramic stacks. The photo of the fabricated twomode shearing and expanding piezoelectric actuators is shown in figure 5 .

In general, a piezoelectric actuator requires a high voltage input to be effectively activated. In the case of the fabricated shearing and expanding piezoelectric actuators, their maximum voltage ratings were $\pm 200 \mathrm{~V}$ and $+100 \mathrm{~V}$, respectively. Therefore, a voltage amplifier is required to amplify the input voltage signals to the piezoelectric actuators without significant distortion. The desired sawtooth and pulse voltage signals were generated in the LabVIEW signal generation system and they were amplified by the piezoamplifier. The piezo-amplifier used in this research was PZD350, which was produced by TREK, Inc., and the specifications are as follows: output voltage range of 0 to $\pm 350 \mathrm{~V}$ in case of $\mathrm{DC}$ or peak $\mathrm{AC}$, maximum output current rating of $\pm 200 \mathrm{~mA}$, maximum DC voltage gain of $150 \mathrm{~V} / \mathrm{V}, \mathrm{DC}$ accuracy better than $0.1 \%$ of full scale with $100 \mathrm{~V} / \mathrm{V}$ gain, slew rate greater than $500 \mathrm{~V} \mu \mathrm{s}^{-1}$, small signal bandwidth $(\sim 3 \mathrm{~dB})$ of DC greater than $100 \mathrm{kHz}$ and large signal bandwidth (1\% distortion) of DC greater than $40 \mathrm{kHz}$.

\subsection{Piezoelectric multi-axis stage}

The 2-DOF stick-and-clamping actuation technology was implemented in the innovative design of the piezoelectric multi-axis stage that is shown in figure 6. As can be seen in figure 6 , three two-mode shearing piezoelectric actuators were located $120^{\circ}$ apart in a circular fashion, and three expanding piezoelectric actuators were also positioned in the same manner. As a result, six piezoelectric actuators were positioned $60^{\circ}$ apart in a circular way. The circular slider was then placed on the tips of three two-mode shearing piezoelectric actuators. After assembling the piezoelectric actuators and circular slider, the ball retainer was separated from the thrust ball bearing and located on the circular slider. Consequently, its balls had direct contact with the upper surface of the slider. The bearing cover was also assembled to the ball retainer to limit its motion in lateral directions with the cover structure. Thus, the thrust 


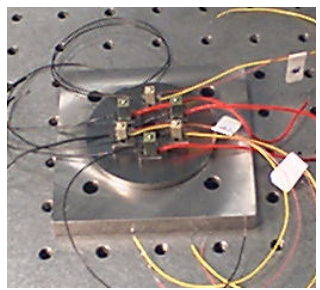

Actuators

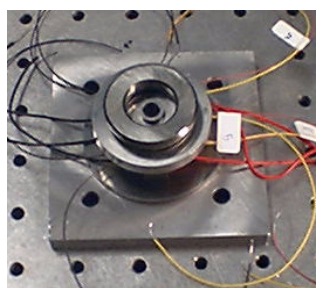

Actuators + Slider +

Bearing

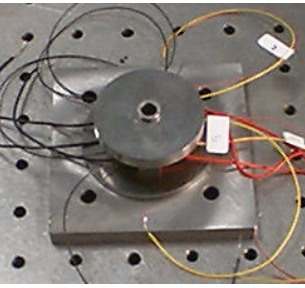

Actuators + Slider

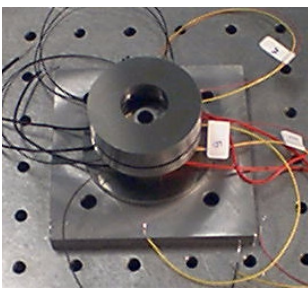

Actuators + Slider +

Bearing + Bearing cover

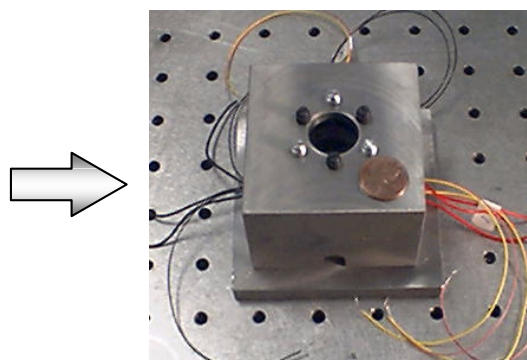

Piezoelectric

multi-axis stage

Figure 7. Photos of the components and assembly of the prototype of the 2-DOF piezoelectric multi-axis stage.

bearing unit did not follow the motion of the slider and could provide the preloads to the slider more effectively. Three spring ball plungers, which were positioned above the three two-mode shearing piezoelectric actuators, were tapped into the cover structure to complete the preload system. The spring ball plunger is a locating device that contains a compressive spring and a spherical stainless steel ball, and the balls of three spring ball plungers had direct contact with the surface of the bearing cover. Therefore, the magnitudes of the preloads applied to the slider could be precisely controlled by tightening and loosening three spring ball plungers. This preload system could control the friction force at each contact interface, resulting in more effective and precise motion of the slider.

Figure 7 shows photos of the fabricated components and their assembly to complete the prototype of the piezoelectric multi-axis stage. In stick mode, only two-mode shearing piezoelectric actuators must have contacts with the slider at its lower surface. Therefore, the gap between the expanding piezoelectric actuators and the lower surface of the slider should be allowed in their deactivated state. The gap distance was less than $4.5 \mu \mathrm{m}$, which was operational displacement of the expanding piezoelectric actuators, and was regulated by using a coordinate measuring machine (CMM) and precision shims.

The material of the various components of the developed piezoelectric multi-axis stage was stainless steel (SS440C). A grinding process was conducted to selected surfaces such as the slider and the surface of the bottom plate to which the piezoelectric actuators are attached for effective actuation. The static preloads applied to the slider were monitored by using three FlexiForce ${ }^{\circledR}$ force sensors, which are piezoresistive force sensors manufactured by Tekscan Incorporated. They were attached to the surface of the bearing cover at the points where the spring ball plungers were located.

The stick-and-clamping actuation technology can provide unlimited travel since the slider is driven by the inchworm actuation principle during its stick mode. Therefore, the maximum travel of the piezoelectric multi-axis stage could be determined by its structural design and was set to be $10 \mathrm{~mm}$.

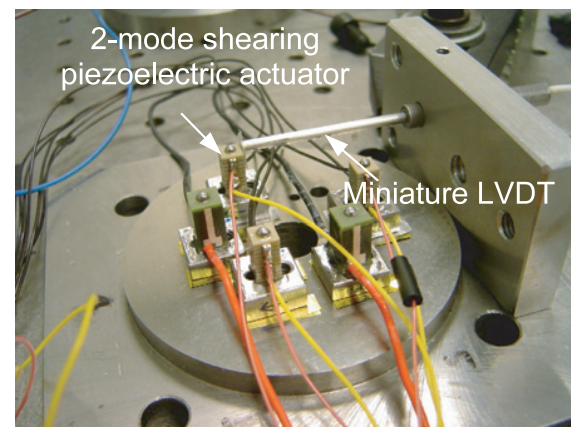

Figure 8. Photo of the displacement evaluation system for the two-mode shearing piezoelectric actuator. The core of the miniature LVDT was attached to the upper side surface of the two-mode shearing piezoelectric actuator.

In addition, the size of the developed prototype was $70 \mathrm{~mm} \times$ $70 \mathrm{~mm} \times 50 \mathrm{~mm}$.

\section{Performance analysis and experimental validation}

\subsection{Performance evaluation of the two-mode shearing piezoelectric actuator}

The displacement performance of the two-mode shearing piezoelectric actuator was experimentally evaluated by using a miniature linear variable differential transducer (LVDT). Figure 8 shows the photo of the displacement evaluation system for the two-mode shearing piezoelectric actuator.

The displacement of the two-mode shearing piezoelectric actuator was measured according to various step voltage inputs. The applied voltages were $\pm 100, \pm 150$, and $\pm 200 \mathrm{~V}$, and the corresponding displacements are summarized in table 1 . The displacements shown in table 1 were averaged values of 10 tests at each voltage input and the results showed good repeatability less than $0.1 \mu \mathrm{m}$. The same displacement performance test was also conducted in the other poling direction, and the measured displacements were identical to those given in table 1. 


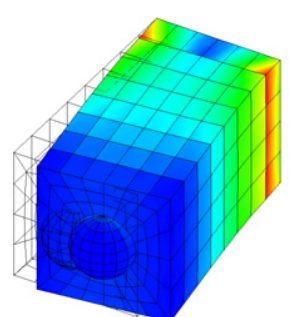

(a)

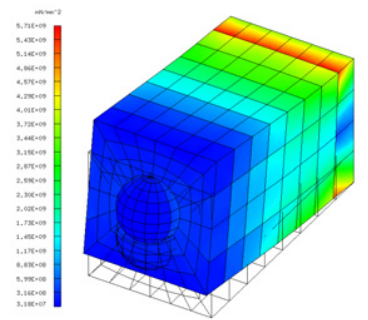

(b)

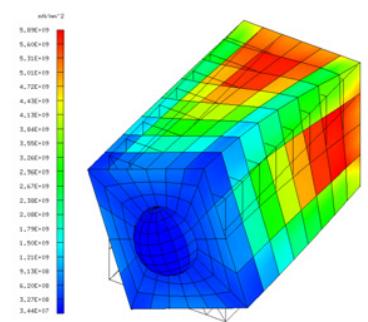

(c)

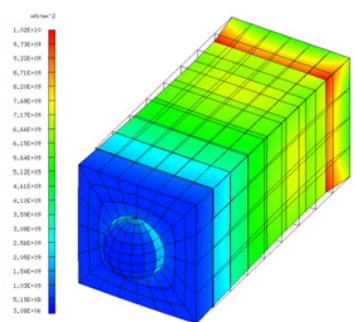

(d)

Figure 9. Natural mode shapes of piezoelectric actuators: (a) first mode, (b) second mode, (c) third mode, (d) fourth mode.

Table 1. Displacements of the two-mode shearing piezoelectric actuator.

\begin{tabular}{lllllll}
\hline Input voltage $(\mathrm{V})$ & -200 & -150 & -100 & 100 & 150 & 200 \\
Displacement $(\mu \mathrm{m})$ & -0.8 & -0.6 & -0.4 & 0.4 & 0.6 & 0.8 \\
\hline
\end{tabular}

The relationship between input voltages and resulting displacements was linear, and the piezoelectric shear mode coefficient $d_{15}$ given in equation (1) could then be simply calculated. Since the shearing piezoelectric actuator that deforms in a single direction was composed of four thin shearing piezoelectric elements, the displacement of each piezoelectric element could be assumed as a quarter of the total displacement of the shearing piezoelectric actuator. The calculated coefficient $d_{15}$ of a single shearing piezoelectric element was $1 \times 10^{-12} \mathrm{~m} \mathrm{~V}^{-1}$.

\subsection{Dynamics of piezoelectric actuators}

The dynamics of piezoelectric actuators could have a significant influence on the selection of the driving frequency of the sawtooth voltage input and the parameters of the pulse voltage input. Therefore, the dynamic behaviors of the piezoelectric actuators were numerically analyzed by generating their finite element models, and the natural modes and their natural frequencies were obtained. The physical and material properties of piezoelectric actuators and stainless steel hemispheres are given in table 2.

It was observed that both piezoelectric actuators had the same order of natural modes from the numerical analysis, and the first four natural mode shapes of the piezoelectric actuators are given in figure 9. As can be seen in figure 9, the first and second natural modes are the first bending modes, and the third natural mode is the first torsional mode. In addition, the fourth natural mode is the longitudinal extension mode. The natural frequencies of each mode for both piezoelectric actuators are also given in table 3. These natural mode behaviors of the piezoelectric actuators could affect the actuation efficiency and system stability, and, therefore, the driving frequency of the sawtooth and pulse voltage input should be carefully chosen.

Due to the maximum current limit of the piezo-amplifier PZD350, which is $\pm 200 \mathrm{~mA}$, the maximum driving frequency was calculated to be $15.1 \mathrm{kHz}$ when considering the total capacitance value of three expanding piezoelectric actuators (2.1 $\mu \mathrm{F})$, which are electronically connected in parallel. The driving frequency less than $15.1 \mathrm{kHz}$ may not be influenced by the resonant dynamics of the two-mode shearing
Table 2. Physical and material properties of the piezoelectric element and hemispherical tip.

\begin{tabular}{lll}
\hline & $\begin{array}{l}\text { Piezoelectric } \\
\text { element } \\
(\text { PIC-255) }\end{array}$ & $\begin{array}{l}\text { Hemispherical } \\
\text { tip } \\
(\text { SS440C) }\end{array}$ \\
\hline Young's modulus $(E)$ & $63 \mathrm{GPa}$ & $200 \mathrm{GPa}$ \\
Poisson's ratio $(v)$ & 0.31 & 0.30 \\
Density $(\rho)$ & $7750 \mathrm{~kg} \mathrm{~m}^{-3}$ & $7800 \mathrm{~kg} \mathrm{~m}^{-3}$ \\
\hline
\end{tabular}

Table 3. Natural frequencies of piezoelectric actuators.

\begin{tabular}{lll}
\hline \multirow{2}{*}{$\begin{array}{l}\text { Natural } \\
\text { modes }\end{array}$} & \multicolumn{2}{c}{ Natural frequency $(\mathrm{kHz})$} \\
\cline { 2 - 3 } & $\begin{array}{l}\text { Two-mode shearing } \\
\text { piezoelectric actuator }\end{array}$ & $\begin{array}{l}\text { Expanding } \\
\text { piezoelectric actuator }\end{array}$ \\
\hline First mode & 25.4 & 23.7 \\
Second mode & 25.9 & 24.0 \\
Third mode & 50.9 & 44.6 \\
Fourth mode & 90.0 & 79.0 \\
\hline
\end{tabular}

and expanding piezoelectric actuators, whose lowest natural frequencies were $25.4 \mathrm{kHz}$ and $23.7 \mathrm{kHz}$, respectively.

\subsection{Dynamics of contact interfaces}

4.3.1. Dynamic model. The study of the dynamic characteristics of the contact interfaces between the hemispherical tips of the shearing piezoelectric actuators and the circular slider is of great significance since the motion of the slider driven by the friction force highly depends on the contact conditions. The study of friction at a contact area between two surfaces has a long history in the fields of mechanics, metallurgy, tribology and control. Several different models for friction behavior and the dynamics of a contact area have been developed by many researchers using both theoretical and experimental approaches [28-31]. The LuGre friction model proposed by Canudas de Wit et al [32] has been used in the contact model since it can provide a better understanding of the friction dynamic behavior of the contact by simply introducing equivalent springs and dampers. The LuGre friction model includes the sticking behavior and the Stribeck effect that represents the decrease in friction in a low velocity domain, and can be used for the dynamic characterization of the stick mode of the stickand-clamping actuation system. The mathematical formula to 
Development of a piezoelectric multi-axis stage based on stick-and-clamping actuation technology

describe the LuGre friction model is given in equation (2):

$$
F=\sigma_{0} z+\sigma_{1} \frac{\mathrm{d} z}{\mathrm{~d} t}+\sigma_{2} v
$$

where $\sigma_{0}$ is the stiffness, $\sigma_{1}$ the damping coefficient, $\sigma_{2}$ the viscous friction coefficient, $v$ the relative velocity between the two surfaces and $z$ the deflection of the bristles. The dynamics of the 1-DOF system driven by the LuGre friction force can be described by equation (3):

$$
m \frac{\mathrm{d}^{2} x}{\mathrm{~d} t^{2}}=-F=-\sigma_{0} z-\sigma_{1} \frac{\mathrm{d} z}{\mathrm{~d} t}-\sigma_{2} \frac{\mathrm{d} x}{\mathrm{~d} t}
$$

where $x$ is the relative displacement between the two surfaces. Since the average deflection of the bristles denoted by $z$ can be modeled by $\frac{\mathrm{d} z}{\mathrm{~d} t}=v-\frac{|v|}{g(v)} z$, where $g(v)$ is the function to describe the Stribeck effect, its linearization in the vicinity of $z=0$ and $v=0$ yields $\frac{\mathrm{d} z}{\mathrm{~d} t}=\frac{\mathrm{d} x}{\mathrm{~d} t}$ and equation (3) becomes

$$
m \frac{\mathrm{d}^{2} x}{\mathrm{~d} t^{2}}+\left(\sigma_{1}+\sigma_{2}\right) \frac{\mathrm{d} x}{\mathrm{~d} t}+\sigma_{0} x=0
$$

Equation (4) describes the friction dynamic behavior in the stick regime and shows that the system behaves like a second-order damped system. The bristle stiffness, $\sigma_{0}$, is usually very large, and thus a non-zero value of $\sigma_{1}$ is necessary to obtain a sufficiently damped motion, since the viscous friction coefficient, $\sigma_{2}$, may not be large enough to provide good damping. From equation (4), therefore, the contact interface between two surfaces can be modeled as an equivalent spring and damper whose coefficients are $\sigma_{0}$ and $\left(\sigma_{1}+\sigma_{2}\right)$, respectively.

The dynamic modeling approach for the contact interface on the basis of the LuGre friction model, which is given in equation (4), was applied to the stick-and-clamping actuation system to describe its stick mode. In the developed piezoelectric multi-axis stage, the slider had contacts with the tips of the two-mode shearing piezoelectric actuators and the balls of the thrust bearing unit at the same time in the stick mode. Figure 10 shows the schematic diagrams of the slider in the stick mode and its 1-DOF equivalent dynamic model based on the LuGre friction model, respectively. The 1-DOF motion of the slider along the $x$ direction was considered in the dynamic analysis.

From the equivalent dynamic model given in figure 10(b), the equation of motion of the slider can be obtained as follows:

$$
M \ddot{x}_{\mathrm{s}}+\left(C_{1}+C_{2}\right) \dot{x}_{\mathrm{s}}+\left(K_{1}+K_{2}\right) x_{\mathrm{s}}=C_{1} \dot{x}_{\mathrm{a}}+K_{1} x_{\mathrm{a}}
$$

where $C_{i}$ is the equivalent damping coefficient, $K_{i}$ the equivalent stiffness, $M$ the mass of the slider, and $x_{\mathrm{s}}$ and $x_{\mathrm{a}}$ are the displacements of the slider and the tips of the twomode shearing piezoelectric actuators, respectively. When equation (5) is compared with equation (4), the sum of damping and viscous friction coefficients are replaced with the total equivalent damping coefficient $C_{i}$ and the stiffness, $\sigma_{0}$, can be replaced with the equivalent stiffness, $K_{i}$. These parameters were identified from the experimental dynamic responses.

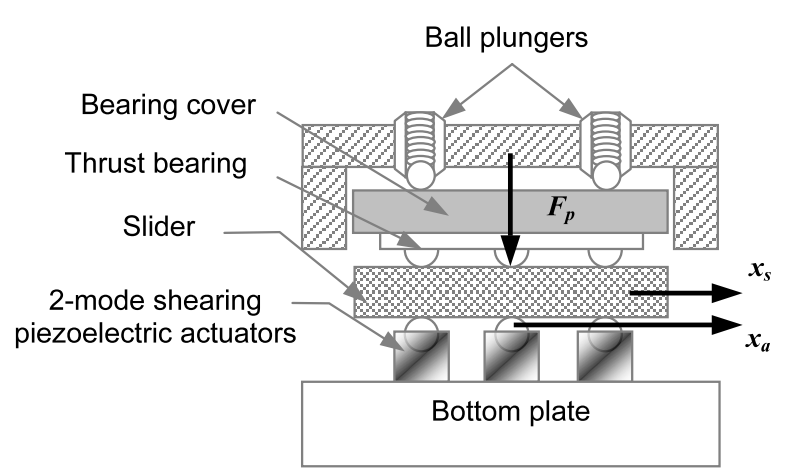

(a)

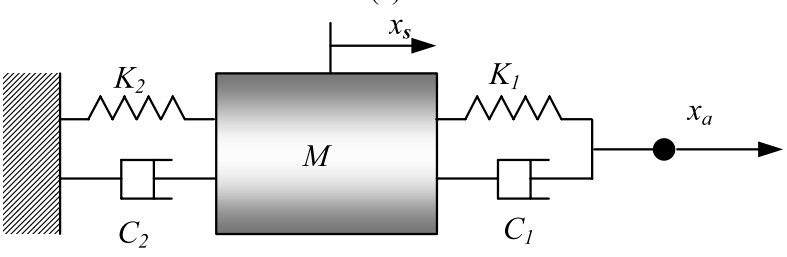

(b)

Figure 10. Schematic diagrams of (a) the slider and (b) its equivalent model during stick mode.

4.3.2. Parameter identification. Equivalent parameters such as stiffness and damping coefficients can be identified from the dynamic responses of the slider when the square voltage inputs are applied to the two-mode shearing piezoelectric actuators. The transfer function corresponding to the ratio of the displacement of the slider to that of the two-mode shearing piezoelectric actuator $\left(X_{\mathrm{s}} / X_{\mathrm{a}}\right)$ was calculated as equation (6) after the Laplace transformation of equation (5):

$$
\begin{aligned}
& \frac{X_{\mathrm{s}}(s)}{X_{\mathrm{a}}(s)}=\frac{C_{1} s+K_{1}}{M s^{2}+\left(C_{1}+C_{2}\right) s+\left(K_{1}+K_{2}\right)} \\
& =\frac{2 \zeta_{1} \omega_{1} s+\omega_{1}^{2}}{s^{2}+2 \zeta_{2} \omega_{2} s+\omega_{2}^{2}}
\end{aligned}
$$

where

$\omega_{1}=\sqrt{\frac{K_{1}}{M}}, \quad \omega_{2}=\sqrt{\frac{K_{1}+K_{2}}{M}}, \quad \zeta_{1}=\frac{1}{2 \omega_{1}} \frac{C_{1}}{M}$,

and $\quad \zeta_{2}=\frac{1}{2 \omega_{2}} \frac{C_{1}+C_{2}}{M}$.

The $\omega_{i}$ is a system natural frequency and $\zeta_{i}$ a damping ratio, respectively.

The experimental system to obtain the dynamic responses of the slider is shown in figure 11. As can be seen in figure 11, the dynamic responses of the slider were measured by the non-contact optical method using a laser module and position-sensitive detectors (PSDs). The laser module included a pigtailed laser diode and a cube beamsplitter, thus generating two perpendicular laser beams. This laser module was attached to the slider with the aid of a steel extension bar, and two perpendicular laser beams from the laser module stroke the surfaces of two PSDs. A PSD is an optoelectronic device that converts an input light spot into corresponding voltage signals which are processed into positional data of the light spot on its active area. Therefore, the motion of the slider can be monitored by capturing PSD positional data. 


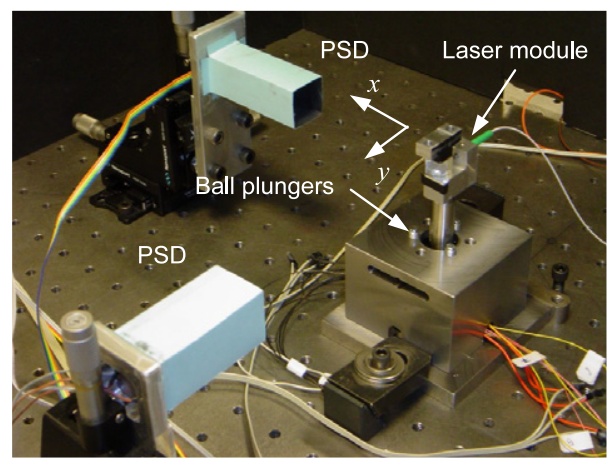

Figure 11. Photo of the experimental system of the 2-DOF piezoelectric multi-axis stage.

Since the maximum voltage input to the two-mode shearing piezoelectric actuator is $\pm 200 \mathrm{~V}$, its maximum displacement is $\pm 0.8 \mu \mathrm{m}$. To utilize the full scale displacement of the two-mode shearing piezoelectric actuator, a square voltage input was applied. The magnitudes of the square voltage signals were $\pm 150 \mathrm{~V}\left(\mathrm{~V}_{\mathrm{pp}}=300 \mathrm{~V}\right)$ and $\pm 190 \mathrm{~V}\left(\mathrm{~V}_{\mathrm{pp}}=380 \mathrm{~V}\right)$, and the total deformations of the two-mode shearing actuators were $1.2 \mu \mathrm{m}$ and $1.5 \mu \mathrm{m}$, respectively. The profiles of the square voltage inputs and corresponding displacements of the two-mode shearing piezoelectric actuators are given in figure 12. The static value of the total preload was set to be $4.5 \mathrm{~N}$, and the anticipated magnitude of the friction force was $2.4 \mathrm{~N}$ by assuming the friction coefficient at the contact interface between the tips of the two-mode shearing piezoelectric actuators and the lower surface of the slider to be 0.4 [28].

The resulting displacements of the slider and the displacement inputs of the two-mode shearing piezoelectric actuators are shown in figure 13. As can be seen in figure 13, the steady state values of the displacements of the slider to each square voltage input were $1.07 \mu \mathrm{m}$ and $1.33 \mu \mathrm{m}$, respectively. These steady state values are smaller than the displacement inputs of the two-mode shearing piezoelectric actuators, which are likely caused by the frictional lag at the contact interfaces. To identify the stiffness and damping coefficients in the

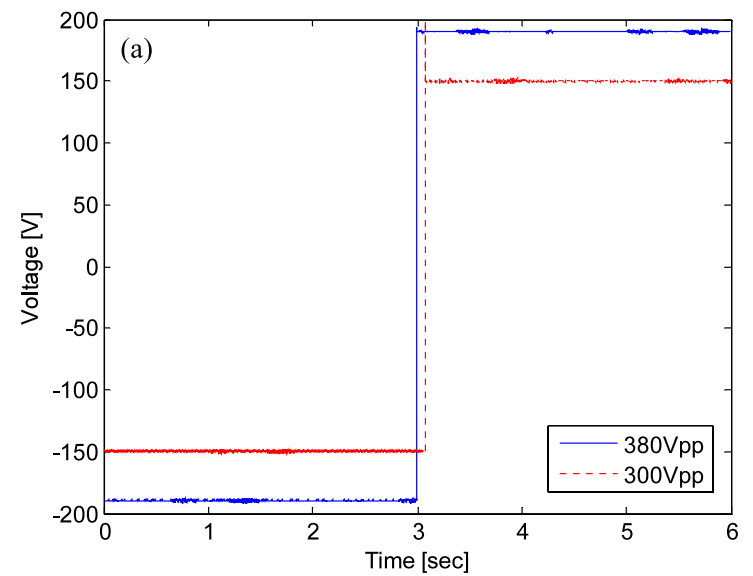

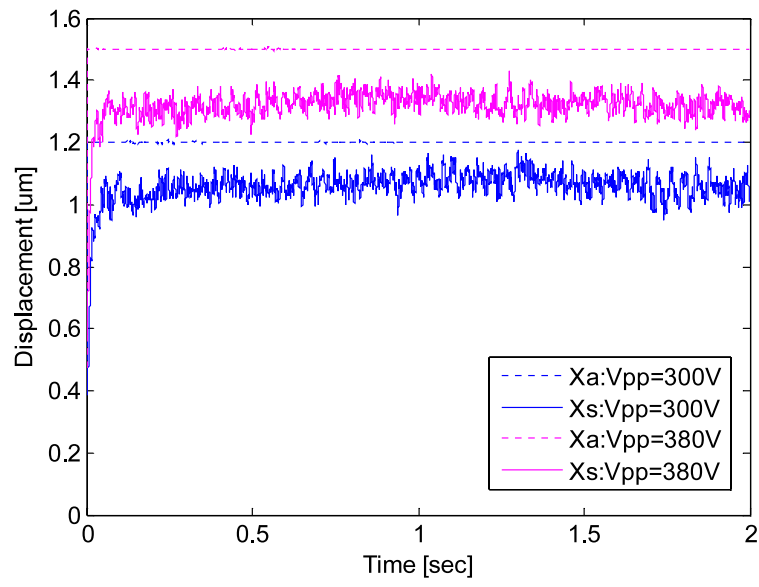

Figure 13. Dynamic responses of the slider versus square voltage inputs.

equivalent model, the ratio of the displacement of the slider to that of the two-mode shearing piezoelectric actuators $\left(x_{\mathrm{s}} / x_{\mathrm{a}}\right)$ was obtained, and these ratios from both cases were identical, which was 0.89 .

The identification process was carried out using experimental dynamic response data. The physical properties of the system and the identified equivalent parameters are summarized in table 4 . Interface 1 represents the contact between the tips of the two-mode shearing piezoelectric actuators and the slider and Interface 2 does the contact between the slider and the thrust ball bearing unit. As can be seen in table 4, the equivalent parameters such as stiffness and damping coefficients in cases 1 and 2 are similar in magnitude. In both cases, the stiffness of Interface 1 was an order of magnitude larger than that of Interface 2. Since the lubrication grease was provided to the balls of the retainer of the thrust bearing unit at Interface 2, the friction coefficient at Interface 2 could be smaller than that of Interface 1 having a dry contact without any lubrication medium. As a result, the magnitude of the friction force at Interface 1 was larger than that at Interface 2, thus allowing the motion of the slider to occur. The reduced friction

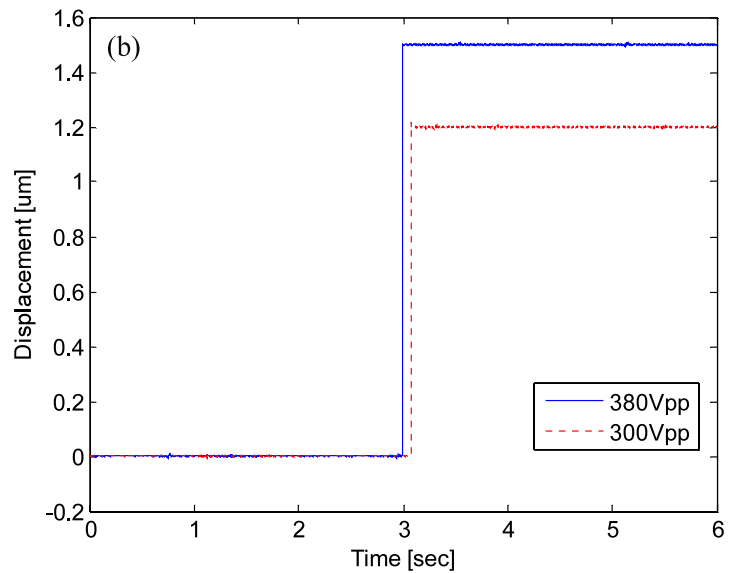

Figure 12. Profiles of (a) square voltage inputs to two-mode shearing piezoelectric actuators and (b) their corresponding step-like displacements in the cases of \pm 150 and $\pm 190 \mathrm{~V}$. By applying square voltage inputs, the magnitudes of the displacements of the two-mode shearing piezoelectric actuators were doubled when compared to the case of step voltage inputs. The rise time of the applied square voltage inputs was measured to be $0.04 \mathrm{~ms}$, and that of the displacements of the shearing piezoelectric actuators was $0.1 \mathrm{~ms}$. 
Development of a piezoelectric multi-axis stage based on stick-and-clamping actuation technology
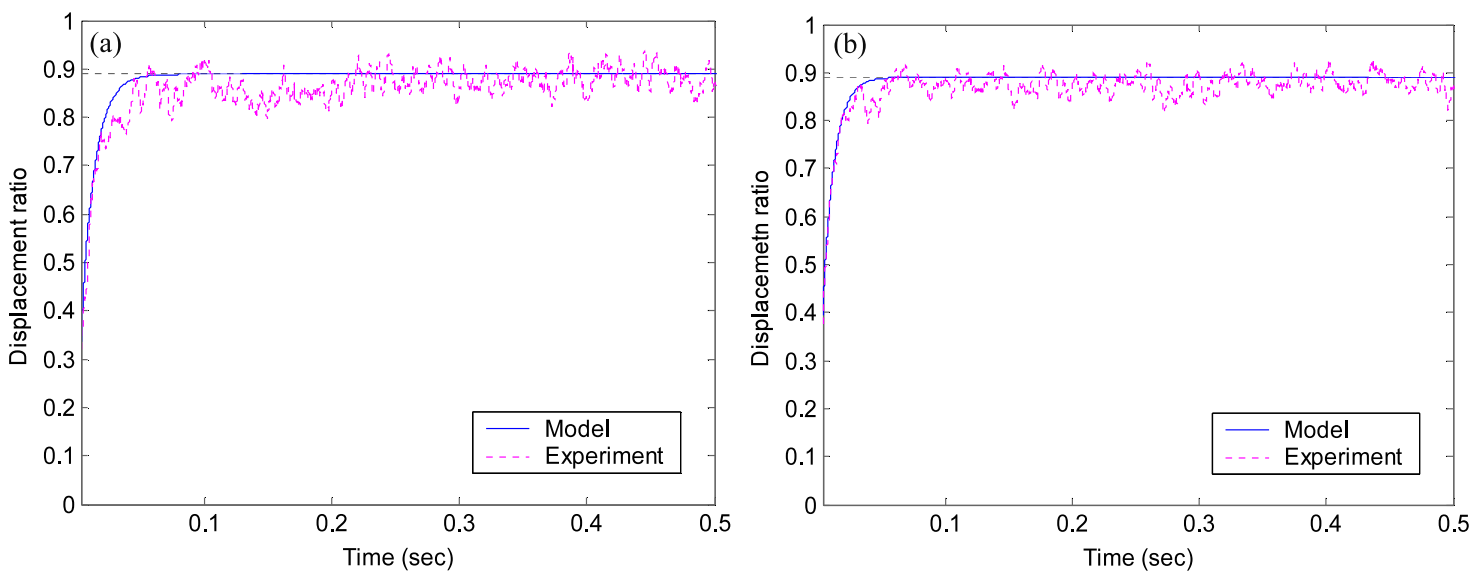

Figure 14. Dynamic responses of the displacement ratios from experiment and model: (a) case 1: $V_{\mathrm{pp}}=300 \mathrm{~V}$, (b) case $2: V_{\mathrm{pp}}=380 \mathrm{~V}$.

Table 4. Physical properties of the system and identified equivalent parameters.

\begin{tabular}{|c|c|c|c|}
\hline & Conta & Interface 1 & Interface 2 \\
\hline & Mass of slider (kg) & \multicolumn{2}{|c|}{$M=0.163$} \\
\hline U্ & $\begin{array}{c}\text { Stiffness }\left(\mathrm{N} \mathrm{m}^{-1}\right) \\
\text { Damping coefficient }\left(\mathrm{N} \mathrm{s} \mathrm{m}^{-1}\right) \\
\text { Damping ratio }\end{array}$ & $\begin{array}{l}K_{1}=1.362 \times 10^{4} \\
C_{1}=44.57 \\
\zeta_{1}=0.4729\end{array}$ & $\begin{array}{l}K_{2}=1.702 \times 10^{3} \\
C_{2}=133.33 \\
\zeta_{2}=1.7798\end{array}$ \\
\hline $\begin{array}{l}\mathcal{N} \\
\tilde{z} \\
\tilde{g} \\
\tilde{J}\end{array}$ & $\begin{array}{c}\text { Stiffness }\left(\mathrm{N} \mathrm{m}^{-1}\right) \\
\text { Damping coefficient }\left(\mathrm{N} \mathrm{s} \mathrm{m}^{-1}\right) \\
\text { Damping ratio }\end{array}$ & $\begin{array}{l}K_{1}=1.562 \times 10^{4} \\
C_{1}=64.73 \\
\zeta_{1}=0.6413\end{array}$ & $\begin{array}{l}K_{2}=1.930 \times 10^{3} \\
C_{2}=115.41 \\
\zeta_{2}=1.6838\end{array}$ \\
\hline
\end{tabular}

force at Interface 2 likely led to a reduction in the stiffness in the equivalent model. However, the opposite phenomena were observed in the case of equivalent damping coefficients. In both cases, the damping coefficients at Interface 2 were larger than those at Interface 1. The lubrication effect at Interface 2 might play a role as an increase in damping that eliminated the overshoot and the oscillatory motion of the slider, and therefore its damping coefficient was larger than that of Interface 1.

Figure 14 shows the dynamic responses of the displacement ratios $\left(x_{\mathrm{s}} / x_{\mathrm{a}}\right)$ from the experiment and model in both cases. As can be seen in figure 14, the dynamic responses of the displacement ratios from the model agree well with those from the experiments in both cases. The transient dynamic behaviors are also very similar in cases 1 and 2 .

\subsection{Open-loop static performance analysis}

The applicability of the proposed piezoelectric multi-axis stage to the mMT should be evaluated in terms of its static performances. Linear, diagonal and circular motion tests were conducted and their open-loop performances were evaluated. In order to verify the advantages of the proposed stick-andclamping actuation over the stick-and-slip actuation for the mMT's positioning system, the motion test results from both actuation technologies were obtained and compared. The experimental system of open-loop static performance analysis was the same as that of the dynamic analysis, which is shown in figure 11 .

4.4.1. 1-DOF linear motion test. To generate 1-DOF linear motion of the slider, the sawtooth voltage signal was applied to one of the two shearing piezoelectric actuators of the two-mode shearing piezoelectric actuator. In addition, the pulse voltage signal was input to the expanding piezoelectric actuator simultaneously to complete the stick-and-clamping actuation. The 1-DOF linear motion tests were conducted in two directions- $x$ and $y$-shown in figure 11. In each linear motion test, two different driving frequencies-500 and $1000 \mathrm{~Hz}$ - of the sawtooth and pulse voltage signals were applied, and the resulting linear displacements were then measured. The frequency of the sawtooth voltage signals to drive the two-mode shearing piezoelectric actuators could control the velocity of the slider. The high frequency of the driving voltage signals results in the high velocity of the slider.

The experimental results of the linear motion tests in the $x$ and $y$ directions are shown in figure 15. The amplitude of the sawtooth voltage signals applied to the two-mode shearing piezoelectric actuators was $\pm 150 \mathrm{~V}\left(300 \mathrm{~V}_{\mathrm{pp}}\right)$ and the corresponding shearing displacement was $\pm 0.6 \mu \mathrm{m}$. The pulse voltage signals to drive the expanding piezoelectric actuators had an amplitude of $75 \mathrm{~V}$ that produced the elongation of $4.5 \mu \mathrm{m}$. The total number of sawtooth shapes was 6000 and, as a result, the total displacement input to the slider from the two-mode shearing piezoelectric actuators was $7.2 \mathrm{~mm}$. The stick-and-slip actuation of the slider could be realized without applying the pulse voltage signals to the expanding piezoelectric actuators and its displacements are also reported in figure 15. The total travel of the slider driven by the stick-and-clamping actuation is larger than that by the stickand-slip actuation. These results confirm the advantage of the proposed stick-and-clamping actuation technology to eliminate the backward motion of the slider in the stick-and- 


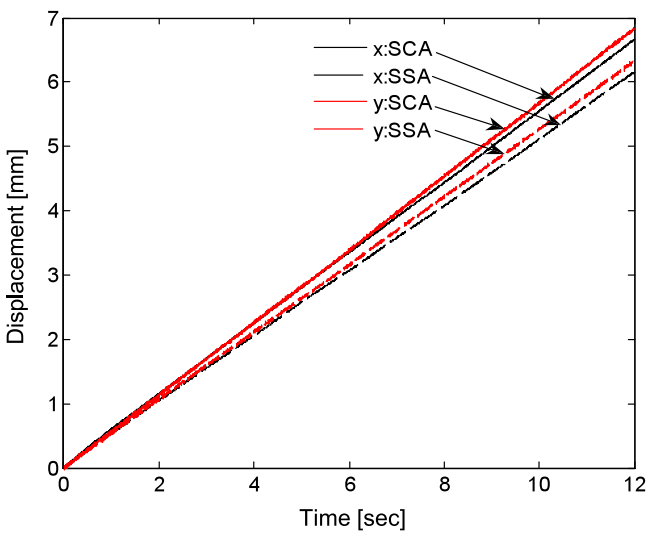

(a)

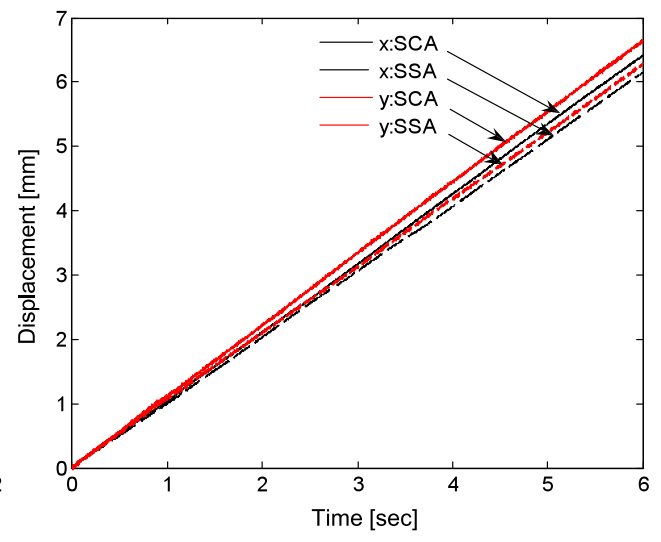

(b)

Figure 15. Linear motions of the slider in the cases of the driving frequencies of (a) $500 \mathrm{~Hz}$ and (b) $1000 \mathrm{~Hz}$ along the $x$ and $y$ directions. SCA stands for stick-and-clamping actuation and SSA stands for stick-and-slip actuation.

Table 5. Measured total travels and average velocities.

\begin{tabular}{|c|c|c|c|c|c|}
\hline & \multirow{2}{*}{$\begin{array}{l}\text { Driving frequency } \\
(\mathrm{Hz})\end{array}$} & \multicolumn{2}{|c|}{ Total travel (mm) } & \multicolumn{2}{|c|}{ Velocity $\left(\mathrm{mm} \mathrm{s}^{-1}\right)$} \\
\hline & & $x$ & $y$ & $x$ & $y$ \\
\hline \multirow[t]{2}{*}{ Stick-and-slip actuation } & 500 & 6.158 & 6.332 & 0.51 & 0.53 \\
\hline & 1000 & 6.146 & 6.300 & 1.02 & 1.05 \\
\hline \multirow[t]{2}{*}{ Stick-and-clamping actuation } & 500 & 6.664 & 6.833 & 0.56 & 0.57 \\
\hline & 1000 & 6.447 & 6.642 & 1.07 & 1.11 \\
\hline
\end{tabular}

slip actuation technology. The constant velocities of the slider were also observed in every case under uniform frequency of the sawtooth voltage signal input.

The total travels and average velocities of the slider from the stick-and-clamping and the stick-and-slip actuation technologies are summarized in table 5. As shown in table 5, the stick-and-clamping actuation system produces longer total travels than those in the stick-and-slip actuation system. However, the increase in the total travel in both $x$ and $y$ directions in the case of $500 \mathrm{~Hz}$ driving frequency-about $0.5 \mathrm{~mm}$ - is larger than that in the case of $1000 \mathrm{~Hz}$ - about $0.3 \mathrm{~mm}$. Due to larger distortion of pulse signals in the piezoamplifier under the condition of higher driving frequency, the clamping action in the case of a $500 \mathrm{~Hz}$ driving frequency could be more effective than that in the case of $1000 \mathrm{~Hz}$, resulting in more total travel at a lower driving frequency.

It is also observed that the total travels of the slider along the $x$ and $y$ directions were not identical despite the same driving voltage inputs for both directions. This difference may be due to nonidentical contact conditions along the $x$ and $y$ directions and misalignment of the two-mode shearing actuators. The average velocity of the slider in the stickand-clamping actuation system was also larger than that in the stick-and-slip actuation system. The velocity of the slider could be controlled by changing the driving frequency and voltage amplitude of the sawtooth signal. The obtained average velocities of the slider in the stick-and-clamping actuation system were applicable to the mMT systems.

Because there was no guideway structure in the piezoelectric multi-axis stage, the lateral and rotational motions of the slider were not limited, and a large crosstalk could occur. The measured crosstalks of the slider during its linear motions along the $x$ and $y$ directions are shown in figure 16 in the cases with and without control of the preloads at the contact interfaces.

These unwanted lateral and rotational motions of the slider were mainly due to misalignment of the two-mode shearing piezoelectric actuators, assembly error of the perpendicularly stacked shearing piezoelectric actuators, non-uniform surface integrity and an unbalanced driving force at each contact interface. In the stick-and-slip actuation system, the preloads to the contact interfaces were provided by the magnetic force or the weight of the slider, and therefore there was no way to control the preloads at each contact interface. On the other hand, in the proposed stick-and-clamping actuation system, three spring ball plungers could precisely control the magnitudes of the preloads and, as a result, the friction forces at each contact interface. Therefore, the crosstalks of the slider were significantly reduced. The preload system in the stickand-clamping actuation system played an important role as a guideway to enhance the crosstalk performances of the slider. As can be seen in figure 16, the preload control can enhance the crosstalk performances of the slider up to approximately $70 \%$.

4.4.2. Diagonal motion test. The diagonal motion of the slider was tested by applying an identical sawtooth voltage signals to two shearing piezoelectric actuators in the two-mode shearing piezoelectric actuator simultaneously. The motion of the slider along the $45^{\circ}$ diagonal line between the $x$ and $y$ directions was then generated. The pulse voltage signal was also applied to the expanding piezoelectric actuators to complete the stick-and-clamping actuation. Figure 17 shows the experimental results of the motion of the slider along both 


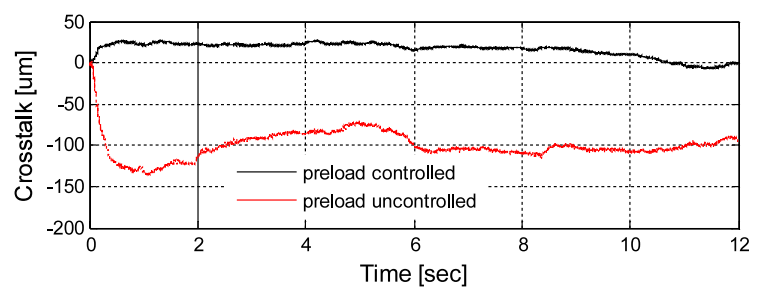

(a)

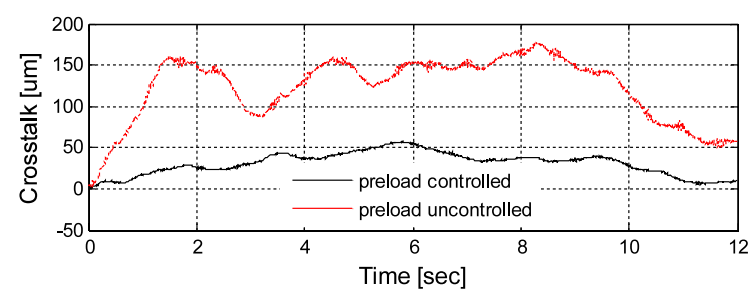

(c)

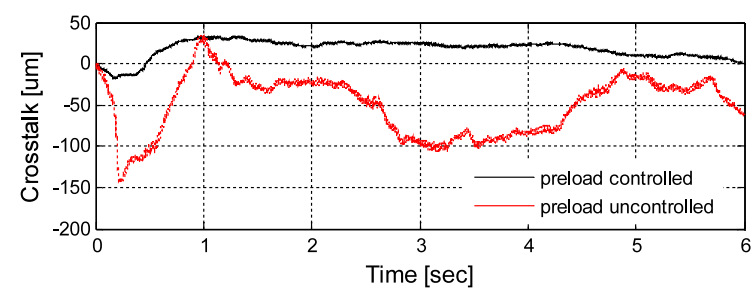

(b)

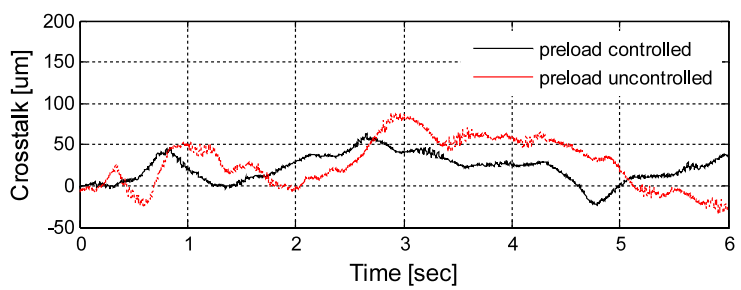

(d)

Figure 16. Crosstalks of the slider during linear motions with driving frequencies of (a) $500 \mathrm{~Hz}$ and (b) $1000 \mathrm{~Hz}$ along the $x$ direction, and (c) $500 \mathrm{~Hz}$ and (d) $1000 \mathrm{~Hz}$ along the $y$ direction.
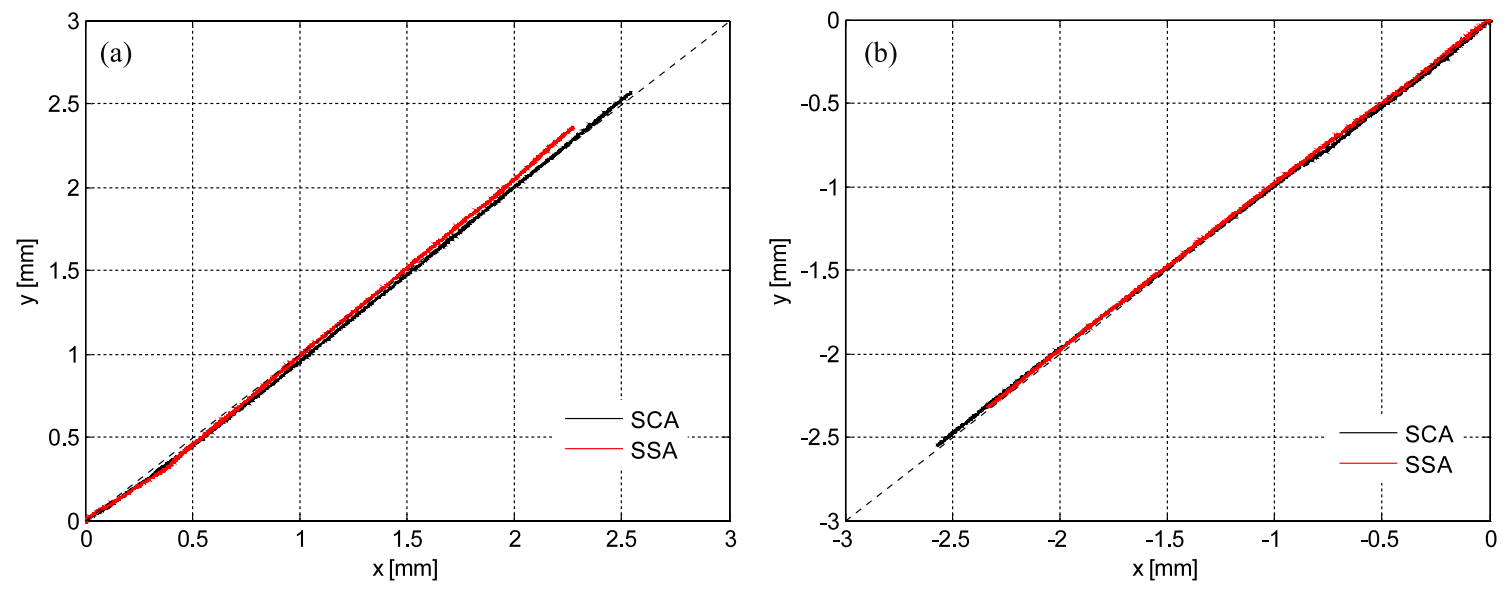

Figure 17. Diagonal motions of the slider along (a) the positive $45^{\circ}$ diagonal direction and (b) the negative $45^{\circ}$ diagonal direction. SCA stands for stick-and-clamping actuation and SSA stands for stick-and-slip actuation.

positive and negative diagonal directions, which was measured by two PSDs. As was done with the 1-DOF linear motion test, the diagonal motions from both the stick-and-clamping actuation and the stick-and-slip actuation were measured and compared to each other.

As can be seen in figure 17 , the total diagonal travels of the slider from the stick-and-clamping actuation are longer than those from the stick-and-slip actuation in both positive and negative directions. The driving frequency and the amplitude of the sawtooth voltage signals to the two-mode shearing piezoelectric actuators were $500 \mathrm{~Hz}$ and $\pm 150 \mathrm{~V}$ $\left(300 \mathrm{~V}_{\mathrm{pp}}\right)$, respectively. In addition, the total number of sawtooth shapes was 2400 , and therefore the total displacement input made by the two-mode shearing piezoelectric actuators to the slider was $\pm 4.07 \mathrm{~mm}$ along the $45^{\circ}$ diagonal line. However, due to the frictional lag at the contact interfaces, the total diagonal displacements of the slider from the stick-andclamping actuation system were $3.614 \mathrm{~mm}$ and $-3.596 \mathrm{~mm}$ in positive and negative diagonal directions, respectively. These diagonal displacements are about $0.3 \mathrm{~mm}$ larger than those

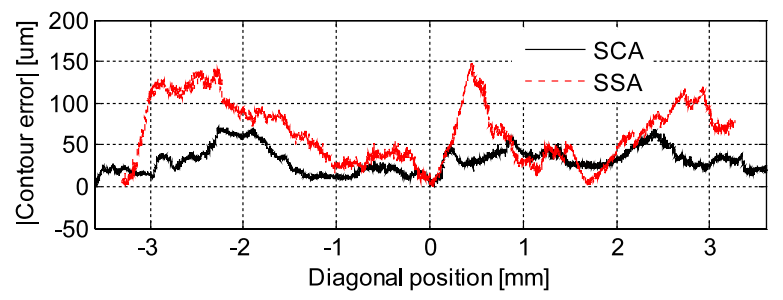

Figure 18. Magnitude of contour errors of diagonal motion of the slider.

from the stick-and-slip actuation system in magnitude, which is a result of the elimination of the backward motion of the slider in the stick-and-clamping actuation system.

The open-loop contour errors of the slider from the $45^{\circ}$ referential diagonal line were also monitored and their results are shown in figure 18. As can be seen in figure 18, the magnitude of the open-loop contour errors of the slider are observed to be less than $75 \mu \mathrm{m}$ in the case of the stick-and- 


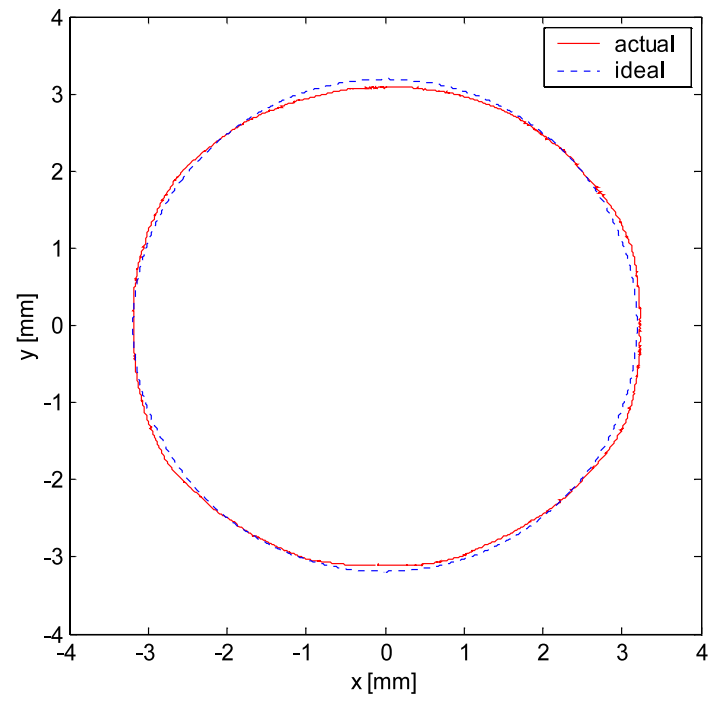

(a)

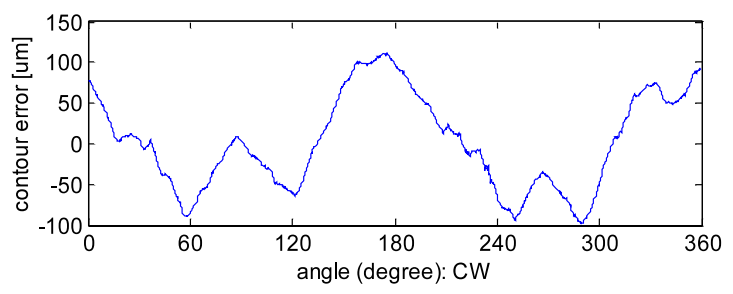

(c)

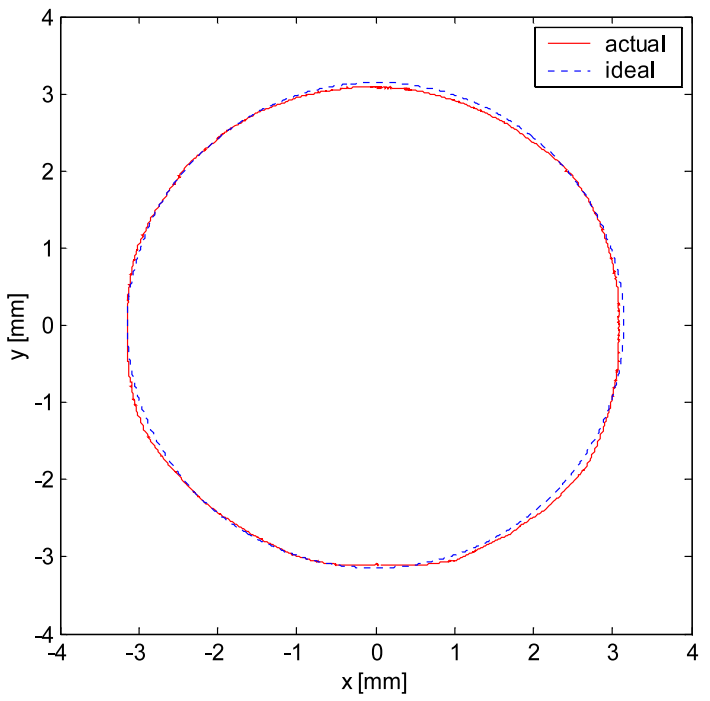

(b)

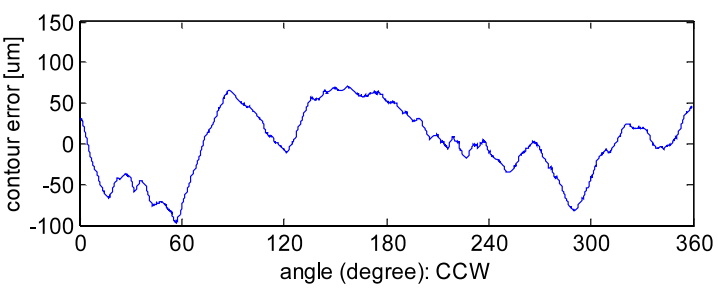

(d)

Figure 19. Circular motions of the slider in (a) clockwise and (b) counterclockwise directions, and contour errors in (c) clockwise and (d) counterclockwise directions.

clamping actuation with control of preloads, which is about only a half of those without preload control in the case of stickand-slip actuation.

The major factors causing contour errors during the diagonal motion of the slider can be summarized as follows:

(1) imperfect perpendicularity of the shearing piezoelectric actuators in the two-mode shearing piezoelectric actuators,

(2) misalignment of three two-mode shearing piezoelectric actuators when assembled to the bottom plate,

(3) degradation of the surface integrity of the slider, and

(4) unsymmetrical actuation of the slider between its 2-DOF directions.

4.4.3. Circular motion test. The circular motion of the slider was generated by applying the voltage signals combining the sawtooth and sinusoidal waves to the two-mode shearing piezoelectric actuators. The sawtooth signals enveloped by sine and cosine waves were applied to each shearing piezoelectric actuator of the two-mode shearing piezoelectric actuators. The pulse voltage signal was also applied to the expanding piezoelectric actuators to complete the stick-andclamping actuation.

The open-loop circular motions of the slider and contour errors were obtained in clockwise and counterclockwise directions, and their results are shown in figure 19. The driving frequency of the sawtooth voltage signal was $500 \mathrm{~Hz}$ and its full voltage amplitude was $\pm 175 \mathrm{~V}\left(350 \mathrm{~V}_{\mathrm{pp}}\right)$. The voltage amplitude of the sawtooth signal was modulated by an enveloping sinusoidal wave, whose frequency was $0.02 \mathrm{~Hz}$. The diameter of the clockwise and counterclockwise circular motions was $6.382 \mathrm{~mm}$ and the total driving time was $50 \mathrm{~s}$. As can be seen in figures 19(a) and (b), the open-loop circular motions of the slider show a very good agreement with the ideal circular profiles in both cases. The contour errors of the open-loop circular motions of the slider were less than $100 \mu \mathrm{m}$, as shown in figures 19(c) and (d). The major factors causing the contouring errors of the slider during its open-loop circular motions are similar to those in the case of the openloop diagonal motions of the slider, which are given in the previous section.

\section{Conclusions}

A new stick-and-clamping actuation technology has been proposed, and its proof-of-concept prototype was developed. The stick-and-clamping actuation system consisted of three two-mode shearing piezoelectric actuators, three expanding piezoelectric actuators, the circular slider and the advanced preload system. There are two operating modes in the stick-and-clamping actuation technology: (1) stick mode and (2) clamp mode. In stick mode, the deformation of the shearing piezoelectric actuators drove the slider by means of the friction force at their contact interfaces under the sawtooth voltage input. On the other hand, in clamp mode, the expanding piezoelectric actuators provide the clamping force to the slider 
Development of a piezoelectric multi-axis stage based on stick-and-clamping actuation technology

and, as a result, eliminate its backward motion following the rapid backward deformation of the shearing piezoelectric actuators. The two-mode shearing piezoelectric actuators, realized by stacking two shearing piezoelectric actuators in a perpendicular fashion, enabled the in-plane 2-DOF motion of the circular slider. The advanced preload system could also minimize unwanted lateral and rotational motions by precisely controlling the preloads at contact interfaces with spring ball plungers and a thrust ball bearing. The proof-of-concept prototype of the 2-DOF piezoelectric multi-axis stage based on the stick-and-clamping actuation technology has been designed and fabricated. The overall size of the developed multi-axis stage was $70 \mathrm{~mm} \times 70 \mathrm{~mm} \times 50 \mathrm{~mm}$, and the maximum travel was $10 \mathrm{~mm}$.

Dynamic analysis was conducted on the stick mode of the stick-and-clamping actuation technology. The LuGre friction modeling approach was introduced and the contact interfaces were modeled by equivalent springs and dampers. The equivalent stiffness and damping coefficients were identified from the experimental dynamic responses of the circular slider. The dry contact at the lower surface of the slider with the tips of the two-mode shearing piezoelectric actuators could be modeled as higher stiffness and lower damping, while the lubricated contact at the upper surface of the slider with the thrust ball bearing could be modeled as lower stiffness and higher damping.

Open-loop static performance analysis was also conducted by generating linear, diagonal and circular motions in the developed 2-DOF multi-axis stage, and those motions were evaluated by the optical measurement system. The motions generated by the proposed stick-and-clamping actuation system had longer travels than those generated by the stickand-slip actuation system through the effective elimination of the backward motion. In addition, the advanced preload system of the stick-and-clamping actuation system could precisely control the preloads to each contact interface, resulting in significant reductions in crosstalks and contour errors of the motions. Therefore, the open-loop static performances of the developed 2-DOF piezoelectric multi-axis stage showed its applicability to the positioning system of the mMT.

\section{Acknowledgments}

This research was conducted at the S M Wu Manufacturing Research Center of the University of Michigan where the corresponding author had stayed for his $\mathrm{PhD}$ study and postdoctoral research fellowship. The authors would like to thank the National Science Foundation for the financial support throughout the research.

\section{References}

[1] Ni J and Mayor R 2003 Meso-scale mechanical machine tools and micro-milling process development for future micro-factory based manufacturing Proc. 7th Int. Conf. on Mechatronics Technology pp 33-9

[2] Stibitz R 1964 Incremental feed mechanisms US Patent $3,138,749$

[3] Hsu S, Arbor A and Blatter A 1966 Transducer US Patent 3,292,019

[4] Locher G L 1967 Micrometric linear actuator US Patent $3,296,467$
[5] Brisbane A D 1968 Position control device US Patent 3,377,489

[6] Galutva G V 1972 Device for precision displacement of a solid body US Patent 3,684,904

[7] Bizzigotti R A 1975 Electromechanical translational apparatus US Patent 3,902,085

[8] May W G 1975 Piezoelectric electromechanical translation apparatus US Patent 3, 902,084

[9] EXFO Burleigh Product Group http://www.exfo.com/en/ burleigh.asp

[10] O'Neill C G and Foster C E 1980 Electromotive actuator US Patent 4,219,755

[11] Taniguchi T 1984 Piezoelectric driving apparatus US Patent 4,454,441

[12] Staufenberg C W and Hubbell R J 1986 Piezoelectric electromechanical translation apparatus US Patent 4,622,483

[13] Fujimoto T 1988 Linear motor driving device US Patent 4,736,131

[14] Murata T 1990 Drive apparatus and motor unit using the same US Patent 4,947,077

[15] Rennex G 1994 Inchworm actuator US Patent 5,332,942

[16] Meisner J E and Teter J P 1994 Piezoelectric/magnetostrictive resonant inchworm motor Proc. SPIE_Smart Struct. Intell. Syst. 2190 520-7

[17] Lee S and Esashi M 1995 Design of the electrostatic linear microactuator based on the inchworm motion Mechatronics 5 963-72

[18] Pandell T and Garcia E 1996 Design of a piezoelectric caterpillar motor Proc. ASME Aerospace Division vol 52 pp 627-48

[19] Galante T, Frank J, Bernard J, Chen W and Lesieutre G 1999 Design, modeling, and performance of a high force piezoelectric inchworm motor J. Intell. Mater. Syst. Struct. 10 962-72

[20] Canfield S, Edinger S, Frecker B M and Koopmann G 1999 Design of piezoelectric inchworm actuator and compliant end-effect for minimally invasive surgery Proc. SPIE-Smart Struct. Integrated Syst. 3668 835-43

[21] Kim J, Kim J D and Choi S B 2002 A hybrid inchworm linear motor Mechatronics 12 525-42

[22] Suleman A, Burns S and Waechter D 2004 Design and modeling of an electrostrictive inchworm actuator Mechatronics 14 567-86

[23] Li J, Sedaghati R, Dargahi J and Waechter D 2005 Design and development of a new piezoelectric linear Inchworm ${ }^{\circledR}$ actuator Mechatronics 15 651-81

[24] Kim J and Kim J-H 2005 Self-moving cell linear motor using piezoelectric stack actuators Smart Mater. Struct. 14 934-40

[25] Breguet J and Clavel R 1998 Stick and slip actuators: design, control, performances and applications Proc. IEEE-Int. Symp. on Micromechatronics and Human Science pp 89-95

[26] Breguet J and Renaud P 1996 A 4-degrees-of-freedom microrobot with nanometer resolution Robotica 14 199-203

[27] Breguet J, Pernette E and Clavel R 1996 Stick and slip actuators and parallel architectures dedicated to microrobotics Proc. SPIE-Microrobotics: Components and Applications 2906 13-24

[28] Rabinowicz E 1995 Friction and Wear of Materials (New York: Wiley)

[29] Courtney J and Eisner E 1957 The effect of a tangential force on the contact of metallic bodies Proc. R. Soc. A 238 529-50

[30] Dahl P R 1977 Measurement of solid friction parameters of ball bearing Proc. 6th Ann. Symp. on Incremental Motion pp 49-60

[31] Swevers J, Al-Bender F, Ganseman C G and Prajogo T 2000 An integrated friction model structure with improved presliding behavior for accurate friction compensation IEEE Trans. Automatic Control 45 675-86

[32] Canudas de Wit C, Olsson H, Åström K J and Lischinsky P 1995 A new model for control of systems with friction IEEE Trans. Automatic Control 40 419-25 